\title{
CHRONIC CEREBROSPINAL VENOUS INSUFFICIENCY THEORY AFTER THE BRAVE DREAMS STUDY
}

\author{
Marian Simka \\ Department of Anatomy, University of Opole, Opole, Poland
}

\author{
EDITORIAL PAPER \\ Phlebological Review 2018; 26 1: 1-3 \\ D0l: https://doi.org/10.5114/pr.2018.76382 \\ Submitted: 20.05 .2018 \\ Accepted: 20.05 .2018 \\ Address for correspondence \\ Marian Simka \\ Department of Anatomy \\ University of Opole \\ 11a Kopernika Pl. \\ 45-040 Opole, Poland \\ e-mail: mariansimka@poczta.onet.pl
}

Multiple sclerosis (MS) is a chronic disease of the central nervous system. It is generally accepted by neurologists that it is an autoimmune disease, which is caused by an attack against nervous tissue antigens, primarily myelin, and that this reaction is executed by reactive $\mathrm{T}$ cells. However, what is actually causing this immune reaction still remains enigmatic. Moreover, there is strong evidence that MS, in addition to its immunoinflammatory aspect, at its core is a neurodegenerative disorder, casting doubt at the autoimmune paradigm [1-3]. Therapeutic strategies for MS predominantly target inflammatory cascade or modify the immune response. These pharmacological treatments are somewhat effective, but they cannot cure patients or protect them from the disability that is usually seen in those with long-lasting MS.

About 10 years ago an Italian vascular surgeon Paolo Zamboni strode into this rather murky milieu with his chronic cerebrospinal venous insufficiency (CCSVI) theory. He demonstrated that MS patients present with haemodynamically significant lesions in the extracranial veins draining the brain, primarily in the internal jugular veins (IJV) - an entity that had not been known before $[4,5]$. He also challenged the autoimmune paradigm, claiming that these venous blockages play a primary role in initiating pathological reactions of MS, including inflammation and chronic cerebral ischaemia. At that time Zamboni and his colleagues also presented results of a pilot study, which showed clinical improvement in MS patients after endovascular balloon angioplasty of pathological IJVs and azygous veins [6].

Consequently, the CCSVI hypothesis began to be hotly debated within the scientific community. Not surprisingly, this new idea was generally welcomed by vascular interventionalists [7] but vigorously challenged by neurologists [8]. During following years a number of open-label studies on endovascular management of CCSVI lesions were published. These treatments usually utilised balloon angioplasty, but in some patients an off-label stenting of the IJVs was also performed. These studies demonstrated the safety of such treatments, especially if balloon angioplasty was used [9-14]. On the other hand, these surveys revealed that endovascular angioplasty in this venous territory is not easy. Technical success after standard balloon angioplasty could be as low as $50 \%$. This rate could be improved using high-pressure balloons and long inflation times, but at the cost of acute thrombosis or subsequent scarring recoil in some patients. Some centres implanted stents if balloon angioplasty alone was not able to restore proper flow. This strategy, which is widely accepted in patients presenting with iliac vein stenosis and is both safe and effective in this area, in the case of abnormal IJVs was often followed by severe intimal hyperplasia, resulting in stent occlusion. In some patients even stent migration to heart chambers or pulmonary arteries occurred. In addition to these technical problems, although the majority of these studies demonstrated clinical improvement after endovascular treatment, all these studies had an open-label design and therefore the placebo effect could not be ruled out. Other researchers reported the presence of CCSVI in patients with non-MS neurological diseases, also in healthy individuals, so this condition did not seem to be exclusively associated with MS, as was initially claimed by Zamboni et al. [15-17]. Finally, analysis of the characteristics of CCSVI lesions has revealed that these venous abnormalities are probably not causing MS, but rather they represent permissive pathology, which can be responsible for clinical progression of MS but not its initiation [18].

The results of a randomised prospective controlled trial on venous angioplasty for CCSVI in MS patients 
were long awaiting by the medical community. Such a trial would definitively answer the question of whether clinical improvements revealed by open-label studies were associated with placebo effect or were indeed related to the procedure itself. Although initially a number of such trials were planned, most of them were not performed, either due to lack of funding or because of problems with recruitment of enough patients to demonstrate statistically significant differences between treatment and sham groups. By 2014 only one trial (USA) had been completed and its results published. However, in this study only a small number of patients were managed, so no valid conclusions could be drawn, except for the safety of the procedure [19]. Other studies that were planned and registered, for example American and Australian trials, were cancelled because doctors were unable to recruit enough patients. In 2017 the results of a Canadian study were revealed. Here researchers did not find different clinical outcomes in patients who underwent angioplasty and those who received only a sham procedure [20]. However, the description of the endovascular technique used by this team suggests that the patients probably received suboptimal treatment, and the lack of improvement could be associated with unsuccessful angioplasties and not the fact that such a procedure was not clinically beneficial. This year the results of a multicentre Italian trial (the Brave Dreams study) were published [21]. This study was planned to involve at least 300 patients, but only 112 completed the study. Similarly to the Canadian trial, the Brave Dreams study has shown that venous jugular angioplasty in MS patients is safe but largely clinically ineffective. Similarly to the former study, a substantial proportion of angioplasties were haemodynamically ineffective. The Italian study has, however, demonstrated that patients who received the treatment had no new MRI-detectable lesions (this composite is routinely used for the assessment of the pharmacological agent used in the treatment of MS). This effect was not statistically significant but could have reached the level of $p<0.05$ if more patents had been recruited $[22,23]$. This effect, however, was seen only in a subgroup of patients.

This year the results of another study were published, which are contradictory to the previous ones. In this study the authors demonstrated improvement after endovascular angioplasty of IJVs in MS patients in terms of relief from headaches and chronic fatigue accompanying MS [24]. Similarly to the Brave Dreams study, this improvement occurred only in a subgroup of patients. Importantly, it was significantly correlated with the technical success of the procedure defined as improved flow after angioplasty.

How do we summarise all these contradictory findings? Not surprisingly, the majority of neurologists concluded that management of malformed veins in MS patients has been proven ineffective and no further research is needed $[25,26]$. Nevertheless, it seems that there are a group of MS patients, primarily those who present with relapsing-remitting course of the disease, especially if MS presents with headaches and severe chronic fatigue, who could potentially benefit from correction of malformed veins. In addition, if in such patients' venous anatomy is favourable, which means that they present with venous stenoses that can easily be corrected using balloon angioplasty alone (it has been demonstrated by many interventionalists that such lesions as stenoses of distal and middle part of the IJV, external compression of these veins, tight stenoses, and long intraluminal flaps respond poorly to balloon angioplasty) [27], these patients could be safely relieved from some troubling symptoms of MS. However, to demonstrate this potentially beneficial effect, another prospective study should be performed [28]. Taking into account how difficult it was to perform the previous studies on this topic (funding, recruitment, approval by authorities, etc.), it is unlikely that such a new study will be conducted.

The author declares no conflict of interest.

\section{References}

1. Chaudhuri A, Behan PO. Multiple sclerosis is not an autoimmune disease. Arch Neurol 2004; 61: 1610-1612.

2. Stys PK. Pathoetiology of multiple sclerosis: are we barking up the wrong tree? F1000Prime Rep 2013; 5: 20.

3. Behan PO. Futility of the autoimmune orthodoxy in multiple sclerosis research. Expert Rev Neurother 2010; 10: 1023-1025.

4. Zamboni P, Galeotti R, Menegatti E, Malagoni AM, Tacconi G, Dall'Ara S, Bartolomei I, Salvi F. Chronic cerebrospinal venous insufficiency in patients with multiple sclerosis. J Neurol Neurosurg Psychiatry 2009; 80: 392-399.

5. Zamboni P, Galeotti R. The chronic cerebrospinal venous insufficiency syndrome. Phlebology 2010; 25: 269-279.

6. Zamboni P, Galeotti R, Menegatti E, Malagoni AM, Gianesini S, Bartolomei I, Mascoli F, Salvi F. A prospective open-label study of endovascular treatment of chronic cerebrospinal venous insufficiency. J Vasc Surg 2009; 50: 1348-1358.

7. Lee BB, Bergan J, Gloviczki P, Laredo J, Loose DA, Mattassi R, Parsi K, Villavicencio JL, Zamboni P, International Union of Phlebology (IUP). Diagnosis and treatment of venous malformations Consensus document of the International Union of Phlebology (IUP)-2009. Int Angiol 2009; 28: 434-451.

8. Doepp F, Paul F, Valdueza JM, Schmierer K, Schreiber SJ. No cerebrocervical venous congestion in patients with multiple sclerosis. Ann Neurol 2010; 68: 173-183.

9. Denislic M, Milosevic Z, Zorc M, Ravnik IZ, Mendiz O. Disability caused by multiple sclerosis is associated with the number of extracranial venous stenoses: possible improvement by venous angioplasty. Results of a prospective study. Phlebology 2013; 28: 353-360.

10. Ludyga T, Kazibudzki M, Simka M, Hartel M, Swierad M, Piegza J, Latacz P, Sedlak L, Tochowicz M. Endovascular treatment for chronic cerebrospinal venous insufficiency: is the procedure safe? Phlebology 2010; 25: 286-295.

11. Lugli M, Morelli M, Guerzoni S, Maleti O. The hypothesis of patho-physiological correlation between chronic cerebrospinal 
venous insufficiency and multiple sclerosis: rationale of treatment. Phlebology 2012; 27 Suppl 1: 178-186.

12. Mandato KD, Hegener PF, Siskin GP, Haskal ZJ, Englander MJ, Garla S, Mitchell N, Reutzel L, Doti C. Safety of endovascular treatment of chronic cerebrospinal venous insufficiency: a report of 240 patients with multiple sclerosis. J Vasc Intervent Radiol 2012; 23: 55-59.

13. Simka M, Janas P, Ludyga T, Latacz P, Kazibudzki M. Endovascular treatment for chronic cerebrospinal venous insufficiency in patients with multiple sclerosis. Vasc Dis Manag 2012; 9: 149-154.

14. Petrov I, Grozdinski L, Kaninski G, Iliev N, Iloska M, Radev A. Safety profile of endovascular treatment for chronic cerebrospinal venous insufficiency in patients with multiple sclerosis. J Endovasc Ther 2011; 18: 314-323.

15. Grozdinski L, Petrov I, Iloska M. Chronic cerebrospinal venous insufficiency in amyotrophic lateral sclerosis. Gazz Med Ital Arch Sci Med 2012; 171: 605-611.

16. Dolic K, Weinstock-Guttman B, Marr K, Valnarov V, Carl E, Hagemeier J, Brooks C, Kilanowski C, Hojnacki D, Ramanathan M, Zivadinov R. Risk factors for chronic cerebrospinal venous insufficiency (CCSVI) in a large cohort of volunteers. PLoS One 2011; 6: e28062.

17. Dolic K, Weinstock-Guttman B, Marr K, Valnarov V, Carl E, Hagemeier J, Kennedy C, Kilanowski C, Hojnacki D, Ramanathan M, Zivadinov R. Heart disease, overweight, and cigarette smoking are associated with increased prevalence of extra-cranial venous abnormalities. Neurol Res 2012; 34: 819-827.

18. Simka M, Ludyga T, Latacz P, Kazibudzki M, Majewski E, Zaniewski M. Chronic cerebrospinal venous insufficiency is unlikely to be a direct trigger of multiple sclerosis. Mult Scler Rel Disord 2013; 2: 334-339.

19. Siddiqui AH, Zivadinov R, Benedict RH, Karmon Y, Yu J, Hartney ML, Marr KL, Valnarov V, Kennedy CL, Ramanathan M, Ramasamy DP, Dolic K, Hojnacki DW, Carl E, Levy EI, Hopkins LN, Weinstock-Guttman B. Prospective randomized trial of venous angioplasty in MS (PREMiSe). Neurology 2014; 83: 441-449.

20. Traboulsee A, Machan L, Girard M, Raymond J, Vosoughi R, Hardy B, Edmond F, Bone J, Gariepy JL, Tam R, Klass D, Isserow S, Rauscher A, Sadovnick AD, Li DK, Illes J, Siskin G. Venoplasty of chronic cerebral spinal venous insufficiency to improve MS patient reported outcomes is not superior to sham treatment at week 2 or week 12. J Neurol Sci 2017; 381 (suppl.): 1066.

21. Zamboni P, Tesio L, Galimberti S, Massacesi L, Salvi F, D’Alessandro R, Cenni P, Galeotti R, Papini D, D’Amico R, Simi S, Valsecchi MG, Filippini G, for the Brave Dreams Research Group. Efficacy and safety of extracranial vein angioplasty in multiple sclerosis. JAMA Neurol 2018; 75: 35-43.

22. Juurlink BH, Bavera PM, Sclafani S, Petrov I, Reid D.B. Brave Dreams: an overestimated study, crippled by recruitment failure and misleading conclusions. Veins Lymphatics 2018; 7: 7340.

23. Rasman A. CCSVI in multiple sclerosis: is it the end? Phlebol Rev 2017; 25: 91-92.

24. Beggs CB, Giaquinta A, Veroux M, De Marco E, Mociskyte D, Veroux P. Mid-term sustained relief from headaches after balloon angioplasty of the internal jugular veins in patients with multiple sclerosis. PLoS ONE 2018; 13: e0191534.

25. Zivadinov R, Weinstock-Guttman B. Multiple sclerosis: Extracranial venous angioplasty is ineffective to treat MS. Nat Rev Neurol 2018; 14: 129-130.
26. Green AJ, Kamel H, Josephson SA. Combating the spread of ineffective medical procedures: a lesson learned from multiple sclerosis. JAMA Neurol 2018; 75: 15-17.

27. Giaquinta A, Beggs CB, Veroux M, De Marco E, Sanzone A, Virgilio $C$, Veroux P. Factors influencing the hemodynamic response to balloon angioplasty in the treatment of outflow anomalies of internal jugular veins. J Vasc Surg Venous Lymphat Disord 2017; 5: 777-788.

28. Zhou D, Ding JY, Ya JY, Pan LQ, Yan F, Yang Q, Ding YC, Ji XM, Meng R. Understanding jugular venous outflow disturbance. CNS Neurosci Ther 2018; 24: 473-482. 